\title{
CONSTRUCTIVE SOLUTION OF COUPLED SECOND ORDER DELAY DIFFERENTIAL EQUATIONS WITH VARIABLE COEFFICIENTS
}

\section{R.J. VILLANUEVA $†$, A. HERVAS $\dagger$ and M.V. FERRER $\ddagger$}

†Departamento do Matemática Aplicada

Émversidad Politécmica de Valencia

PO Box 22012, Valencıa, Spain

†Dpto de Matemátıcas e Informátıca

Unuv Jaune I. ('ampus de Penyeta Rot.ja

('astellón. Sipaun

(Received August 3, 1993 and in revised form September 13, 1993)

\begin{abstract}
In this paper, we study initial value problems for coupled second order delay differential equations with variable coefficients. By means of the application of the method of stcps and the method of Frobenius, the exact solution of the problem is constructed. Then, in a bounded domain, a finite analytic solution with error bounds is provided. Given an admissible error c, we give the number of terms to be taken in the infinite series exact solution so that the approximation error be smaller than $\epsilon$ in the bounded domain.
\end{abstract}

KEY WORDS AND PHRASES. Delay equations, Variable coefficients.

1991 AMS(MOS) SUBJECT CLASSIFICATION. 34K99, 34A25, 34A05, 65Q05, 65G99.

\section{INTRODUCTION.}

In many fields of the contemporary science and technology systems with delaying links are often met and the dynamical processes in these are described by systems of delay differential eruations, Bellman \& Cooke [1], Driver [3], Marchuck [11], Okamoto \& Hayashi [13]. The delay appears in complicated systems with logical and computing devices, where certain time for information processing is needed.

The theory of linear delay differential equations has been developed in the fundamental monographs Bellman \& Cooke [1], Driver [3], Hale [10], Myshkis [12], Pinney [15]. Analytic solutions of some linear systems of delay differential equations have been investigated by Cherepennikov [2], Jódar \& Martín [8], Jódar \& Martín [9], Rodinov [16].

In this paper, we consider initial value problems for systems of second order delay differential equations of the form

$$
\left.\begin{array}{c}
X^{\prime \prime}(t)+A(t) X^{\prime}(t)+B(t) X(t)+B_{1}(t) X^{\prime}(t-w)=F(t), t>0 \\
X(t)=G(t), t \in[-w, 0], w>0
\end{array}\right\}
$$

where $A(t)$ and $B(t)$ are analytic $\mathcal{C}^{r \times r}$ valued functions on the positive real line, $B_{1}(t)$ is a $\mathcal{C}^{1 \times 1}$ valued continuous function, the unknown $X(t)$ as well as $F(t)$ and $G(t)$ are $\mathcal{C}^{r}$ valued functions, with $F(t)$ continuous in $t>0$ and $G(t)$ is a continuously differentiable function in $[-w, 0]$.

Problem (1.1) can be transformed into the equivalent extended first order system

$$
Z(t)=\left[\begin{array}{c}
X(t) \\
X^{\prime}(t)
\end{array}\right]
$$




$$
\begin{aligned}
& \left.Z^{\prime}(I)+\left[\begin{array}{cc}
0 & 0 \\
0 & B_{1}(t)
\end{array}\right] Z(I-u)+\left[\begin{array}{cc}
0 & -I \\
B(I) & A(I)
\end{array}\right]+Z(I)=\left[\begin{array}{c}
0 \\
V(t)
\end{array}\right], I>0\right) \\
& Z(1)=\left[\begin{array}{l}
(i(1) \\
\left(i^{\prime}(1)\right.
\end{array}\right] .1 \in\left[-u^{\prime} \cdot 0\right]
\end{aligned}
$$

but this approach has some drawbacks, such as the increase of the computational cost and the lack of explicitness due to the relationship $\mathcal{X}(t)=[I, 0] Z(t)$.

The aim of this paper is twofold. First of all we construct a series solution of problem (1.I) by means of a matrix method of Frobenius and the method of steps, but dealing directly with (1.1). Secondly we tunede the series solution and provide enror bounds for the continume linite approximate solution when $t \in[n w,(n+1) w]$ and $n$ is a positive integer. For the constant coeflicient rase. ustems of second order delav differential equations have been recently studied in bóda $\mathbb{d}$ : Mattin [8] and fólar d: Martín [9] avoiding the transformation of the problem into an cquitalent extended first onder system.

This paper is organized as follows. In section 2 we construct a series solution of problem (1.1). Brror analysis of the finite truncated series in terms of the data, for a given interval $[n w,(n+1) u]$, is studied in section 3.

If $P$ is a matrix in $\mathcal{C}^{p \times q}$, we denote by $\|P\|$ the 2 -norm of $P$ defined in Golub \& Van Loan [5, p. 14]

\section{2. $\Lambda$ SERIES SOLUTION OF THE PROBLEM.}

We begin this section considering the differential system

$$
X^{\prime \prime}(t)+A(t) \cdot X^{\prime}(t)+B(t) X(t)=0 .
$$

Let us suppose that $A(t), B(t)$ are $\mathcal{C}^{r \times r}$ valued analytic functions in $|t|<a$ with $0<a \leq+\infty$ and

$$
A(t)=\sum_{n \geq 0} A_{n} t^{n}, B(t)=\sum_{n \geq 0} B_{n} t^{n},|t|<a,
$$

where $A_{n}, B_{n}$ arc matrices in $\mathcal{C}^{r \times r}$. From the Cauchy inequalities, there exists a positive constant L. such that

$$
\left\|A_{n}\right\| \rho^{n} \leq L,\left\|B_{n}\right\| \rho^{n} \leq L, 0<\rho<a, n \geq 0 .
$$

Let us look for $\mathcal{C}^{r \times r}$ solutions of $(2.1)$ of the form $X(t)=\sum_{n \geq 0} C_{n} t^{n}$, where $C_{n}$ is a matrix in $\mathcal{C}^{r \times r}$ to be determined. Assuming the convergence of $X(t)$ and of its formal derivatives

$$
X^{\prime}(t)=\sum_{n \geq 0}(n+1) C_{n+1} t^{n}, X^{\prime \prime}(t)=\sum_{n \geq 0}(n+2)(n+1) C_{n+2} t^{n}
$$

and substituting the expressions into (2.1), it follows that the coefficients $C_{n}$ must satisfy

$$
\sum_{n \geq 0}\left\{(n+2)(n+1) C_{n+2}+\left(\sum_{\jmath=0}^{n}(j+1) A_{n-\jmath} C_{\jmath+1}+B_{n-\jmath} C_{\jmath}\right)\right\} t^{n}=0 .
$$

Equating to zero the coefficient of each power $t^{n}$, one gets

$$
(n+2)(n+1) C_{n+2}=-\sum_{\jmath=0}^{n}\left((j+1) A_{n-\jmath} C_{\jmath+1}+B_{n-}, C_{\jmath}\right), n \geq 0
$$

where $C_{0}, C_{1}$ are arbitrary matrices in $\mathcal{C}^{r \times r}$. Taking norms in (2.1) and using (2.3). it follows that 


$$
\begin{aligned}
& (n+2)(n+1)\left\|C_{n+2}\right\| \leq \\
& \leq \sum_{l=0}^{n}\left((\jmath+1)\left\|A_{n-\jmath}\right\|\left\|C^{*}{ }_{j+1}\right\|+\left\|B_{n-\jmath}\right\|\left\|C^{*},\right\|\right) \leq \sum_{\jmath=0}^{n}\left(\frac{(\jmath+1)}{\rho^{n-j}} L\left\|C_{\jmath+1}\right\|+\frac{L}{\rho^{n-j}}\left\|C_{\jmath}\right\|\right) \leq \\
& \leq \frac{L}{\rho^{n}} \sum_{j=0}^{n}\left((\jmath+1)\left\|C_{1+1}^{v}\right\|+\left\|C^{\prime},\right\|\right) \rho^{j}+L\left\|C_{n+1}^{v}\right\| \rho .
\end{aligned}
$$

Wo have added the last term for the sake of later convenience. Now, let us introduce the sequence of positive numbers $\left\{\gamma_{n}\right\}_{n \geq 0}$ defined by $\gamma_{0}=\left\|C_{0}\right\|, \gamma_{1}=\left\|C_{1}\right\|$, and for $n \geq 0, \gamma_{n+2}$ is defined by the recurrent equation

$$
(n+2)(n+1) \gamma_{n+2}=\frac{L}{\rho^{n}} \sum_{j=0}^{n}\left((j+1) \gamma_{\jmath+1}+\gamma_{\jmath}\right) \rho^{j}+L \gamma_{n+1} \rho, n \geq 0
$$

Hence,

$$
\left\|C_{n}\right\| \leq \gamma_{n}, n \geq 0 .
$$

For $n \geq 1$, we may write (2.5) in the form

$$
n(n+1) \gamma_{n+1}=\frac{L}{\rho^{n-1}} \sum_{\jmath=0}^{n-1}\left((\jmath+1) \gamma_{\jmath+1}+\gamma_{\jmath}\right) \rho^{\jmath}+L \gamma_{n} \rho
$$

and for $n \geq 2$,

$$
\begin{gathered}
\rho n(n+1) \gamma_{n+1}= \\
=\frac{L}{\rho^{n-2}} \sum_{\jmath=0}^{n-2}\left((\jmath+1) \gamma_{\jmath+1}+\gamma_{\jmath}\right) \rho^{\jmath}+L \rho\left(n \gamma_{n}+\gamma_{n-1}\right)+L \gamma_{n} \rho^{2}= \\
=n(n-1) \gamma_{n}+L n \gamma_{n} \rho+L \gamma_{n} \rho^{2},
\end{gathered}
$$

by virtue of (2.6).

Hence,

$$
\gamma_{n+1}=\frac{\left[n(n-1)+L n \rho+L \rho^{2}\right] \gamma_{n}}{\rho(n+1) n}, n \geq 2
$$

and

$$
\lim _{n \rightarrow+\infty} \frac{\gamma_{n+1}|t|^{n+1}}{\gamma_{n}|t|^{n}}=\lim _{n \rightarrow+\infty} \frac{\left[n(n-1)+L n \rho+L \rho^{2}\right] \gamma_{n}}{\rho(n+1) n \gamma_{n}}|t|=\frac{|t|}{\rho}, \rho \in(0, a) .
$$

Thus for any pair of starting matrices $C_{0}$ and $C_{1}$, the series $X(t)=\sum_{n \geq 0} C_{n} t^{n}$ with matrices $C_{n}$ defined by (2.4) is absolutely convergent in $|t|<a$.

Let us denote by $X_{1}(t)$ the solution of (2.1) constructed by the above procedure with $C_{n}$ defined by (2.4) with $C_{0}=I, C_{1}=0$, and let $X_{2}(t)$ be defined in the same way with $C_{0}=0, C_{1}=I$. Then, from Lemma 1 of Jódar \& Legua [7] and the definition given in Jódar \& Legua [7], the pair $\left\{X_{1}(t), X_{2}(t)\right\}$ is a fundamental set of solutions of $(2.1)$ in $|t|<a$, and the set of all $\mathcal{C}^{r}$ solutions of (2.1) in $|t|<a$, is given by

$$
X(t)=X_{1}(t) C+X_{2}(t) D, C, D \in \mathcal{C}^{r} .
$$

Let $H(t)$ be a continuous $\mathcal{C}^{r}$ function in $|t|<a$, and let us consider the non-homogeneous problem

$$
X^{\prime \prime}(t)+A(t) X^{\prime}(t)+B(t) X(t)=H(t),|t|<a .
$$

From Lemma 1 of Jódar \& Legua [7], the $\mathcal{C}^{2 r \times 2 r}$ valued function $W(t)$ defined by 


$$
W(t)=\left[\begin{array}{ll}
X_{1}(1) & X_{2}(1) \\
X_{1}^{\prime}(t) & X_{2}^{\prime}(1)
\end{array}\right]
$$

is invertible in $|t|<a$. Let us denote by

$$
W^{\prime-1}(t)=V^{\prime}(t)=\left[\begin{array}{ll}
V_{11}(t) & V_{12}(t) \\
V_{21}(t) & V_{22}(t)
\end{array}\right], V_{\imath \jmath}(t) \in \mathcal{C}^{\prime x_{1}} .1 \leq 1, J \leq 2 .
$$

Let us look for a particular solution of (2.7) of the form

$$
Z(t)=X_{1}(t) D_{1}(t)+X_{2}(t) D_{2}(t),
$$

where $\left\{X_{1}(t), X_{2}(t)\right\}$ is the above fundamental set of solutions of $(2.1)$ and $D_{1}(t), D_{2}(t)$ are $\mathcal{C}^{\prime}$ valued functions satisfying

$$
W(t)\left[\begin{array}{c}
D_{1}^{\prime}(t) \\
D_{2}^{\prime}(t)
\end{array}\right]=\left[\begin{array}{c}
0 \\
H(t)
\end{array}\right] \cdot|t|<a
$$

Note that $(2.10)$ means that

$$
\begin{gathered}
X_{1}(t) D_{1}^{\prime}(t)+X_{2}(l) D_{2}^{\prime}(t)=0, \\
X_{1}^{\prime}(t) D_{1}^{\prime}(t)+X_{2}^{\prime}(t) D_{2}^{\prime}(l)=H(t) .
\end{gathered}
$$

Hence $Z(t)$ satisfies

$$
\begin{gathered}
Z^{\prime \prime}(t)+A(t) Z^{\prime}(t)+B(t) Z(t)= \\
=\left[X_{1}^{\prime \prime}(t)+A(t) X_{1}^{\prime}+B(t) X_{1}(t)\right] D_{1}(t)+ \\
+\left[X_{2}^{\prime \prime}(t)+A(t) X_{2}^{\prime}+B(t) X_{2}(t)\right] D_{2}(t)+H(t)=H(t), \\
Z(0)=D_{1}(0), Z^{\prime}(0)=D_{2}(0) .
\end{gathered}
$$

A solution of (2.10) for $D_{1}(t), D_{2}(t)$, satisfying $D_{1}(0)=D_{2}(0)=0$ is given by

$$
D_{1}(t)=\int_{0}^{t} V_{12}(x) H(x) d x, D_{2}(t)=\int_{0}^{t} V_{22}(x) H(x) d x .
$$

Since (2.7) is linear, its general solution is given by

$$
\begin{gathered}
X(t)=X_{1}(t) C_{1}+X_{2}(t) C_{2}+Z(t)= \\
=X_{1}(t)\left[C_{1}+\int_{0}^{t} V_{12}(x) H(x) d x\right]+X_{2}(t)\left[C_{2}+\int_{0}^{t} V_{22}(x) H(x) d x\right]
\end{gathered}
$$

where $C_{1}, C_{2}$ are arbitrary vectors in $\mathcal{C}^{r}$. Given fixed initial conditions

$$
X(0)=G_{1}, X_{0}^{\prime}=G_{2},
$$

the unique solution of (2.7) satisfying (2.12) is given by (2.11), where the vectors $C_{1}, C_{2}$ must satisfy $G_{1}=X(0)=C_{1}$ and $G_{2}=X^{\prime}(0)=C_{2}$. Thus the following result has been established.

THEOREM 1. Let us consider equation (2.7) where $A(t), B(t)$ are analytic $\mathcal{C}^{r \times r}$ valued functions with power expansions defined by $(2.2)$ and let $H(t)$ be a continuous vector function. Let $\left\{X_{1}(t), X_{2}(t)\right\}$ be the pair of $\mathcal{C}^{r \times r}$ analytic solutions of $(2.1)$ satisfying $X_{1}(0)=I, X_{1}^{\prime}(0)=0$, $X_{2}(0)=0, X_{2}^{\prime}(0)=I$, and let $W(t)$ and $V(t)$ be the $\mathcal{C}^{2 r \times 2 r}$ valued functions defined by $(2.8)$ and (2.9) respectively: Then, the unique solution of the initial value prob!em (2.7). (2.12), is given by (2.11) where $C_{1}=G_{1}, C_{2}=G_{2}$.

Let us consider the delay differential system (1.1) written in the form 


$$
\left.\begin{array}{c}
X^{\prime \prime}(t)+A(t) X^{\prime}(t)+B(t) X(t)=F(t)-B_{1}(t) X^{\prime}\left(t-w^{\prime}\right), t>0 \\
X(t)=G(t), t \in[-w, 0]
\end{array}\right\}
$$

where $A(t), B(t)$ are analytic $\mathcal{C}^{r \times r}$ valued functions on the real line, and note that for $t \in[0, w]$, the right-hand side of (2.13) is a known continuous function. Let $\left\{X_{1}(t), X_{2}(t)\right\}$ be the fundamental set of solutions of (2.1) on the real line provided by Theorem 1, let $W(t)$ and $V(t)$ be defined by Theorem 1 and let us introduce the matrix kernel $K: \mathcal{R} \times \mathcal{R} \longrightarrow \mathcal{C}^{r \times r}$, by the expression

$$
K(t, x)=X_{1}(t) V_{12}(x)+X_{2}(t) V_{22}(x)
$$

and note that from the definition of $V(t)$ and $W(t)$ one gets $K(t, t)=0$ for $t \in \mathcal{R}$.

Let $H(x)$ be a continuous $\mathcal{C}^{\prime}$ valued function and let $K(t, x)$ be defined by $(2.14)$, then we introduce the operator $\mathcal{W}$ defined by

$$
(\mathcal{W} H)(t)=\int_{0}^{t} K(t, x) H(x) d x, t \geq 0 .
$$

It is clear that $\mathcal{W}$ is a linear operator, i.e.,

$$
\mathcal{W}(\alpha H+\beta J)=\alpha \mathcal{W} H+\beta \mathcal{W} J
$$

IIere and below, when possible, we will drop the dependence on $t$ for the sake of brevity.

From Theorem 1, the solution of (2.13) can be written in the form,

$$
X=\mathcal{X}^{\prime}+\mathcal{W}\left(F-B_{1} X_{-w}^{\prime}\right)=\mathcal{X}^{\prime}+\mathcal{W} F-\mathcal{W}\left(B_{1} X_{-w}^{\prime}\right),
$$

where

$$
X_{-w}^{\prime}(x)=X^{\prime}(x-w)
$$

and

$$
\mathcal{X}(t)=X_{1}(t) G(0)+X_{2}(t) G^{\prime}(0), t \geq 0 .
$$

Note that (2.16) is a feedback expression that provides the solution of (1.1) in an interval of length $w$, in terms of the solution in the previous intervals of length $w$. In order to find a closed form expression for the solution of $(1.1)$ in any interval $[n w,(n+1) w]$. we introduce a recurrent sequence of integral operators. If $H$ is a continuously differentiable function in $[-w,+\infty[$, we define for a positive integer $k$,

$$
\mathcal{W}_{1}\left(B_{1} H\right)=\mathcal{W}\left(B_{1} H^{\prime}\right)
$$

and for $k>1$,

$$
\begin{gathered}
\left(\mathcal{W}_{k}\left(B_{1} H\right)\right)(t)=\mathcal{W}\left(B_{1}\left(\mathcal{W}_{k-1}^{\prime}\left(B_{1} H\right)\right)_{-w}\right)(t)= \\
=\int_{0}^{t} K(t, x) B_{1}(x)\left(\mathcal{W}_{k-1}^{\prime}\left(B_{1} H\right)\right)(x-w) d x .
\end{gathered}
$$

As above, $\left(\mathcal{W}_{k-1}^{\prime}\left(B_{1} H\right)\right)_{-w}(t)=\frac{\partial}{\partial t}\left(\mathcal{W}_{k-1}\left(B_{1} H\right)\right)(t-w)$. Now, we prove that the solution of (1.1) can be written in the compact form

$$
X(t)=G(t), t \in[-w, 0]
$$

and for $t \in\left[n u^{\prime},(n+1) u\right]$

$$
\begin{gathered}
X=\mathcal{X}^{\prime}+\mathcal{W} F+\sum_{k=1}^{n}(-1)^{k} \mathcal{W}_{k}\left(B_{1}(\mathcal{W} F)_{-u^{\prime}}++\right. \\
+\sum_{k=1}^{n}(-1)^{k} \mathcal{W}_{k}\left(B_{1} \mathcal{X}_{-w}\right)+(-1)^{n+1} \mathcal{W}_{n+1}\left(B_{1} C_{-w}\right)
\end{gathered}
$$


Indeed, if $t \in[0, w]$, from $(2.16)$ and $(2.18)$ it, follows that

$$
X=\mathcal{X}+\mathcal{W}\left(F-B_{1} G_{-u}^{\prime \prime}\right)=\mathcal{X}+\mathcal{W} F-\mathcal{W}_{1}\left(B_{1} G_{-w}\right) .
$$

Thus (2.20) holds for $n=0$. Let us suppose that (2.20) is true for $t \in[n w,(n+1) w]$ and let. us take $t \in[(n+1) w,(n+2) w]$. From $(2.16)$ and the induction hypothesis, we can write

$$
\begin{gathered}
X=\mathcal{X}+\mathcal{W}\left(F-B_{1} X_{-w}^{\prime}\right)= \\
=\mathcal{X}+\mathcal{W}\left(F-B_{1} \frac{\partial}{\partial t}\left[\mathcal{X}_{-w}+(\mathcal{W} F)_{-w}+\sum_{k=1}^{n}(-1)^{k}\left(\mathcal{W}_{k}\left(B_{1}(\mathcal{W} F)_{-w}\right)\right)_{-w}+\right.\right. \\
\left.\left.+\sum_{k=1}^{n}(-1)^{k}\left(\mathcal{W}_{k}\left(B_{1} \mathcal{X}_{-w}\right)\right)_{-w}+(-1)^{n+1}\left(\mathcal{W}_{n+1}\left(B_{1} G_{-w}\right)\right)_{-w}\right]\right)= \\
=\mathcal{X}+\mathcal{W} F-\mathcal{W}\left(B_{1} \mathcal{X}_{-w}^{\prime}\right)-\mathcal{W}\left(B_{1}(\mathcal{W} F)_{-w}^{\prime}\right)+\sum_{k=1}^{n}(-1)^{k+1} \mathcal{W}\left(B_{1}\left(\mathcal{W}_{k}^{\prime}\left(B_{1}(\mathcal{W} F)_{-w}\right)\right)_{-w}\right)+ \\
+\sum_{k=1}^{n}(-1)^{k+1} \mathcal{W}\left(B_{1}\left(\mathcal{W}_{k}^{\prime}\left(B_{1} \mathcal{X}_{-w}\right)\right)_{-w}\right)+(-1)^{n+2} \mathcal{W}\left(B_{1}\left(\mathcal{W}_{n+1}^{\prime}\left(B_{1} G_{-w}\right)\right)_{-w}\right)= \\
=\mathcal{X}+\mathcal{W} F+\sum_{k=1}^{n+1}(-1)^{k} \mathcal{W}_{k}\left(B_{1}(\mathcal{W} F)_{-w}\right)+ \\
+\sum_{k=1}^{n+1}(-1)^{k} \mathcal{W}_{k}\left(B_{1} \mathcal{X}_{-w}\right)+(-1)^{n+2} \mathcal{W}_{n+2}\left(B_{1} G_{-w}\right) .
\end{gathered}
$$

Note that (2.21) coincides with (2.20) replacing $n$ by $n+1$. Thus the following result has been proved.

THEOREM 2. Let us consider the problem (1.1) under the hypothesis of Theorem 1. Let $\left\{\mathcal{W}_{k}\right\}_{k \geq 1}$ be the sequence of operators defined by $(2.18)$ and $(2.19)$ where $B_{1}(t)$ is a $\mathcal{C}^{r \times r}$ valued continuous function and let $K(t, x)$ be defined by $(2.14)$. If $\mathcal{X}(t)$ is defined by $(2.17)$, then the exact solution of $(1.1)$ in the interval $[n w,(n+1) w]$, for $n \geq 0$, is given by $(2.20)$.

REMARK. It is easy to show that the integral operators $\mathcal{W}_{k}$ defined by $(2.18),(2.19)$ can be written in terms of the data in the form

$$
\begin{gathered}
\left(\mathcal{W}_{k}\left(B_{1} H\right)\right)(t)= \\
=\int_{0}^{t} \int_{0}^{t_{n}-w} \cdots \int_{0}^{t_{p+1}-w}\left[K\left(t, t_{n}\right) B_{1}\left(t_{n}\right) \frac{\partial}{\partial t_{n}} K\left(t_{n}-w, t_{n-1}\right) \cdots\right. \\
\left.\cdots B_{1}\left(t_{p+1}\right) \frac{\partial}{\partial t_{p+1}} K\left(t_{p+1}-w, t_{p}\right) B_{1}\left(t_{p}\right) H\left(t_{p}\right)\right] d t_{p} d t_{p+1} \cdots d t_{n},
\end{gathered}
$$

where $p=n-k+1$.

From a computational point of view, the solution provided by Theorem 2 has the drawback that the expression of $K(t, x)$ and $\mathcal{X}(t)$ is given in terms of infinite series involving $X_{1}(t), X_{2}(t)$ and the block entries of $V(t)$ defined by Theorem 1 . In the sequel we construct finite approximate solutions of (1.1) obtained by truncation of the quoted infinite series.

\section{FINITE ANALYTIC APPROXIMATE SOLUTIONS AND ERROR BOUNDS.}

Let $X_{1}(t)$ and $X_{2}(t)$ be the pair of $\mathcal{C}^{r \times r}$ analytic series solutions of $(2.1)$ given by Theorem 1, let $\imath \geq 1$ and let

$$
\begin{gathered}
X_{1}(t)=\sum_{n=0}^{+\infty} C_{n} t^{n}, X_{2}(t)=\sum_{n=0}^{+\infty} D_{n} t^{n}, 0<t<+\infty, \\
X_{1 \imath}(t)=\sum_{n=0}^{t} C_{n} t^{n}, X_{2 \imath}(t)=\sum_{n=0}^{\imath} D_{n} t^{n},
\end{gathered}
$$




$$
\begin{gathered}
\mathfrak{X}_{1}^{\prime}(t)=X_{12}(t) C_{i}^{\prime}(0)+X_{2 \imath}(t) C_{i}^{\prime}(0), \\
K_{\imath}(t, r)=X_{12}(t) V_{12}^{\prime 2}(x)+X_{2 \imath}(t) V_{22}^{\prime}(t),
\end{gathered}
$$

where $V_{12}^{2}(x)$ and $V_{22}^{2}(x)$ denote the block entries of the inverse of the i-th partial sum of $I I(r)$. (Sice below (3.9)-(3.12)).

Note that from Lemma 1 of Jódar \& Legua [7], the matrix function $W(t)$ defuned by (2.s') satisfies

$$
W^{\prime}(t)=C(t) W(t), W(0)=I_{2 r}, C(t)=\left[\begin{array}{cc}
0 & I \\
-B(t) & -A(t)
\end{array}\right] .
$$

Let $S_{q}$ be the positive constant

$$
S_{q}=\operatorname{cxp}\left(\int_{0}^{(q+1) u^{\prime}}\|C(x)\| d x\right)
$$

From Flett $[4$, p.114], it follows that

$$
\|W(t)\| \leq S_{q},\left\|W^{-1}(t)\right\|=\|V(t)\| \leq S_{q}, t \in[0,(q+1) w]
$$

and

$$
\begin{gathered}
\max \left\{\left\|X_{1}(t)\right\|,\left\|X_{1}^{\prime}(t)\right\|,\left\|X_{2}(t)\right\|,\left\|X_{2}^{\prime}(t)\right\|,\left\|V_{12}(t)\right\|,\left\|V_{22}(t)\right\|\right\} \leq S_{q}, \\
t \in[0,(q+1) w] .
\end{gathered}
$$

From the Cauchy inequalities, it follows that

$$
\left\|C_{n}\right\| \leq S_{q}^{\prime}[z(q+1) w]^{-1},\left\|D_{n}\right\| \leq S_{q}[z(q+1) w]^{-1} . n \geq 1, t \in[0,(q+1) w]
$$

$z$ and $r$ be the positive constants defined by

$$
r=z(q+1) w, z>1
$$

Then, for $0 \leq t \leq(q+1) w$, it follows that

$$
\begin{gathered}
\left\|X_{1}(t)-X_{1 \imath}(t)\right\|=\left\|\sum_{n=\imath+1}^{+\infty} C_{n} t^{n}\right\| \leq \sum_{n=\imath+1}^{+\infty}\left\|C_{n}\right\||t|^{n} \leq \\
\leq S_{q} \sum_{n=\imath+1}^{+\infty}|t|^{n}[z(q+1) w]^{-n}=S_{q} \frac{[(q+1) w]^{i+1}}{[r-(q+1) w] r^{i}}=\frac{S_{q}}{z^{i}(z-1)}=E_{\imath q} .
\end{gathered}
$$

Analogously,

$$
\begin{gathered}
\left\|X_{2}(t)-X_{2 \imath}(t)\right\| \leq \frac{S_{q}}{z^{2}(z-1)}=E_{\imath q} . \\
\left\|X_{1}^{\prime}(t)-X_{1 \imath}^{\prime}(t)\right\|=\left\|\sum_{n=\imath}^{+\infty} n C_{n} t^{n-1}\right\| \leq \sum_{n=\imath}^{+\infty} n\left\|C_{n}\right\||t|^{n-1} \leq \\
\leq \sum_{n=\imath}^{+\infty} n \frac{S_{q}}{r^{n}}|t|^{n-1}=\frac{S_{q}}{r} \sum_{n=\imath}^{+\infty} n\left(\frac{|t|}{r}\right)^{n-1} \leq \frac{S_{q}}{r} \sum_{n=\imath}^{+\infty} n\left(\frac{(q+1) w}{r}\right)^{n-1} \leq \\
\leq \frac{S_{q}}{r}\left[\frac{\imath z}{(z-1) z^{i-1}}+\frac{z^{2}}{(z-1)^{2} z^{i}}\right]=\frac{S_{q}(z-1)(\imath+z-1)}{(q+1) w z^{i-1}}=D_{\imath q}, \\
t \in[0,(q+1) w], \\
\left\|X_{2}^{\prime}(t)-X_{2 \imath}^{\prime}(t)\right\| \leq D_{\imath q}, t \in[0,(q+1) w],
\end{gathered}
$$

where 


$$
D_{\imath q}=E_{\imath q}\left[\frac{z(z-1)^{2}(\imath+z-1)}{(q+1) w}\right]=\frac{S_{q}(z-1)(i+z-1)}{(q+1) w z^{i-1}} .
$$

From (2.17), (3.1), (3.4), (3.5) and (3.6), it follows that

$$
\begin{gathered}
\left\|\mathcal{X}(t)-\mathcal{X}_{\imath}(t)\right\| \leq\left(\left\|G^{\prime}(0)\right\|+\left\|C^{\prime \prime}(0)\right\|\right) E_{\imath q}, t \in[0,(q+1) w] . \\
\|\mathcal{X}(t)\| \leq\left(\left\|G^{\prime}(0)\right\|+\left\|C^{\prime \prime}(0)\right\|\right) S_{q}^{\prime}, t \in[0,(q+1) w] .
\end{gathered}
$$

Since $\left\{E_{\imath q}\right\}$ and $\left\{D_{\imath q}\right\}$ defined by (3.5) and (3.7) respectively, converge to zero as $\rightarrow+\infty$. let us choose $\iota_{0}$ as the first positive integer satisfying

$$
E_{\imath q}+D_{\imath q}<\left(2 S_{q}\right)^{-1}, 1 \geq \imath_{0}
$$

If we denote by $W_{2}(t)$ the $i-t h$ partial sum of $W(t)$, from the porturbation Lemma Orlega [14. p.32] and the inequality

$$
\left\|W(t)-W_{\imath}(t)\right\| \leq 2\left(E_{\imath q}+D_{\imath q}\right)<S_{q}^{-1}<\left\|W^{-1}(t)\right\|^{-1},
$$

it follows that $W_{\imath}(t)$ is invertible and from the Banach Lemma Ortega [14, p.32]

$$
\begin{gathered}
\left\|W^{-1}(t)\right\| \leq S_{q}\left[1-2 S_{q}\left(E_{\imath q}+D_{\imath q}\right)\right]=M_{\imath q}, \\
\left\|W^{-1}(t)-W_{\imath}^{-1}(t)\right\| \leq 2 S_{q}\left(E_{\imath q}+D_{\imath q}\right) M_{\imath q} . t \in[0,(q+1) w],
\end{gathered}
$$

where

$$
W_{\imath}^{-1}(t)=\left[\begin{array}{ll}
V_{11}^{2}(t) & V_{12}^{2}(t) \\
V_{21}^{2}(t) & V_{22}^{2}(t)
\end{array}\right] .
$$

Thus the approximate kernel $K_{\imath}(t, x)$ given by (3.2) for $i \geq i_{0}$, is well defined for $0 \leq t \leq(q+1) w$, in the sense that $W_{\imath}(t)$ is invertible for $i \geq i_{0}$. Then, we can define

$$
\begin{gathered}
\left(\mathcal{W}^{\imath} H\right)(t)=\int_{0}^{t} K_{\imath}(t, x) H(x) d x, t \geq 0, \imath \geq \imath_{0}, \\
\mathcal{W}_{1 \imath}\left(B_{1} H\right)=\mathcal{W}^{\imath}\left(B_{1} H^{\prime}\right), \\
\left(\mathcal{W}_{k \mathfrak{\imath}}\left(B_{1} H\right)\right)(t)=\mathcal{W}^{\imath}\left(B_{1}\left(\mathcal{W}_{k-1, \imath}^{\prime}\left(B_{1} H\right)\right)_{-w}\right)(t)= \\
=\int_{0}^{t} K_{\imath}(t, x) B_{1}(x)\left(\mathcal{W}_{k-1, \imath}^{\prime}\left(B_{1} H\right)\right)(x-w) d x, k>1 .
\end{gathered}
$$

In accordance with (2.22) we can write for $k=n-p+1$

$$
\begin{gathered}
\left(\mathcal{W}_{k, \imath}\left(B_{1} H\right)\right)(t)= \\
=\int_{0}^{t} \int_{0}^{t_{n}-w} \cdots \int_{0}^{t_{p+1}-w}\left[K_{\imath}\left(t, t_{n}\right) B_{1}\left(t_{n}\right) \frac{\partial}{\partial t_{n}} K_{\imath}\left(t_{n}-w, t_{n-1}\right) \cdots\right. \\
\left.\cdots B_{1}\left(t_{p+1}\right) \frac{\partial}{\partial t_{p+1}} K_{\imath}\left(t_{p+1}-w, t_{p}\right) B_{1}\left(t_{p}\right) H\left(t_{p}\right)\right] d t_{p} d t_{p+1} \cdots d t_{n} .
\end{gathered}
$$

Note that from (3.3), (3.9) and the triangular inequality, it follows that

$$
\begin{gathered}
\left\|W_{\imath}(t)\right\| \leq\|W(t)\|+\left\|W(t)-W_{\imath}(t)\right\| \leq S_{q}+S_{q}^{-1}, \\
t \in[0,(q+1) w], i \geq \imath_{0} .
\end{gathered}
$$

From (3.15) and taking into account that 


$$
W_{\imath}(t)=\left[\begin{array}{ll}
X_{12}(t) & X_{2 \imath}(t) \\
X_{12}^{\prime}(t) & X_{2 \imath}^{\prime}(t)
\end{array}\right]
$$

if $1 \geq{ }_{10} .0 \leq 1 \leq(q+1) w$, it follows that

$$
\max \left\{\left\|X_{12}(t)\right\|,\left\|X_{12}^{\prime}(t)\right\|,\left\|X_{21}(t)\right\|,\left\|X_{21}^{\prime}(t)\right\|\right\} \leq S_{q}+S_{q}^{-1} .
$$

Olherwise, note that from the definition of $K(t, x)$ and $K_{\imath}(1, x)$ given by $(2.11)$ and (3.2) respertivel. and from (3.1) and (3.16). it follows that

$$
\begin{aligned}
& \left\|\mathfrak{x}_{\imath}(l)\right\| \leq\left(\left\|C_{i}(0)\right\|+\left\|C^{\prime}(0)\right\|\right)\left[\dot{s}_{q}+i_{\eta}^{\prime-1}\right] . \\
& \left\|K^{\prime}(t, r)-K_{\imath}(I, r)\right\| \leq \gamma_{\imath \eta}=1 S_{q}^{2}\left(E_{\imath q}+D_{\imath q}\right) . M_{\imath \eta}+2 E_{\imath q} M_{\imath q} . \\
& \|K(t, r)\| \leq 2 S_{q}^{2} .\left\|K_{\imath}(t, x)\right\| \leq 2 \cdot M_{\iota q}\left[S_{q}^{\prime}+S_{q}^{-1}\right], \\
& \left\|\frac{\prime \prime}{\partial t} K(t, r)\right\| \leq 2 S_{q}^{\prime 2},\left\|\frac{\prime}{\partial t} K_{2}(t, x)\right\| \leq 2 M_{2 q}\left(S_{q}^{\prime}+S_{q}^{\prime-1}\right) . \\
& \left\|\frac{1}{\prime \prime} K(l, x)-\frac{\prime \prime}{1 t} K_{\imath}(t, x)\right\| \leq 3_{\imath q}=1 . S_{q}^{\prime 2}\left(E_{\imath q}+D_{\imath q}\right) M_{\imath q}+2 D_{\imath q} \cdot M_{\imath \eta} \text {. } \\
& t \in[0,(q+1) w], x \in[0,(q+1) w], 1 \geq i_{0} .
\end{aligned}
$$

Lot, us introduce the constants $g, f_{q}, b_{q}$ defined by

$$
\begin{gathered}
\max \{\|C(t)\|,-w \leq t \leq 0\}=g, \max \{\|F(t)\|, 0 \leq t \leq(q+1) w\}=f_{q}, \\
\max \left\{\left\|B_{1}(t)\right\|, 0 \leq t \leq(q+1) w\right\}=b_{q} .
\end{gathered}
$$

From Gradshtevn [6, p.620], it follows that

$$
\int_{0}^{t-w} \int_{0}^{t_{n}-w} \cdots \int_{0}^{t_{p+1}-w} d t_{p} d t_{p+1} \cdots d t_{n}=\frac{(t-w)[t-(n-p+2) w]^{n-p}}{(n-p+1) !}
$$

In particular. for $t=(n+3) w$ we have

$$
\int_{0}^{(n+2) u} \int_{0}^{t_{n}-w} \cdots \int_{0}^{t_{p+1}-w} d t_{p} d t_{p+1} \cdots d t_{n}=\frac{(n+2)(p+1) u^{n-p+1}}{(n-p+1) !}=I_{n p} .
$$

From (2.22) and (3.14) if $k=n-p+1, t=t_{n+1}$ and $p \in \mathcal{N}$, we can write

$$
\begin{gathered}
\left(\mathcal{W}_{k}\left(B_{1} H\right)\right)(t)-\left(\mathcal{W}_{k \imath}\left(B_{1} T\right)\right)(t)= \\
=\int_{0}^{t} \int_{0}^{t_{n}-w} \cdots \int_{0}^{t_{p+1}-w}\left(\left\{\left[K\left(t, t_{n}\right)-K_{\imath}\left(t, t_{n}\right)\right] B_{1}\left(t_{n}\right)\right.\right. \\
\left.\frac{\partial}{\partial t_{n}} K_{\imath}\left(t_{n}-w, t_{n-1}\right) \cdots B_{1}\left(t_{p+1}\right) \frac{\partial}{\partial t_{p+1}} K_{\imath}\left(t_{p+1}-w, t_{p}\right) B_{1}\left(t_{p}\right) T\left(t_{p}\right)\right\}+ \\
+\sum_{\jmath=p}^{n}\left\{K\left(t, t_{n}\right) B_{1}\left(t_{n}\right) \frac{\partial}{\partial t_{n}} K\left(t_{n}-w, t_{n-1}\right) \cdots\right. \\
\cdots B_{1}\left(t_{\jmath}\right)\left[\frac{\partial}{\partial t_{j}} K\left(t_{\jmath}-w \cdot t_{\jmath-1}\right)-\frac{\partial}{\partial t_{\jmath}} K_{\imath}\left(t_{\jmath}-w, t_{\jmath-1}\right)\right] B_{1}\left(t_{\jmath-1}\right) \cdots \\
\left.\cdots B_{1}\left(t_{p+1}\right) \frac{\partial}{\partial t_{p+1}} K_{\imath}\left(t_{p+1}-w, t_{p}\right) B_{1}\left(t_{p}\right) T\left(t_{p}\right)\right\}+ \\
+\left\{K\left(t, t_{n}\right) B_{1}\left(t_{n}\right) \frac{\partial}{\partial t_{n}} K\left(t_{n_{\imath}}-w, t_{n-1}\right) \cdots B_{1}\left(t_{p+1}\right) \frac{\partial}{\partial t_{p+1}} K^{\prime}\left(t_{p+1}-w, t_{p}\right)\right. \\
\left.\left.B_{1}\left(t_{p}\right)\left[H\left(t_{i}\right)-T\left(t_{p}\right)\right]\right\}\right) d t_{p} d t_{p+1} \cdots d t_{n} .
\end{gathered}
$$


If we denote by $n_{1}$ and $\alpha_{2}$ the constants

$$
\begin{gathered}
\alpha_{1}=\max \left\{\left\|T\left(t_{p}\right)\right\|, p w \leq t_{p} \leq(p+1) w\right\}, \\
\alpha_{2}=\max \left\{\left\|H\left(t_{p}\right)-T\left(t_{p}\right)\right\|, p w \leq t_{p} \leq(p+1) w\right\},
\end{gathered}
$$

from (3.18)-(3.20) it follows that

$$
\begin{gathered}
\left\|\mathcal{W}_{k}\left(B_{1} H\right)-\mathcal{W}_{k \imath}\left(B_{1} T\right)\right\| \leq \\
\leq 2^{n-p+1} I_{n p}\left(\prod_{h=p}^{n} b_{h}\right)\left[\alpha_{1} \gamma_{\imath, n+1}\left(\prod_{h=p}^{n}\left(S_{h}+S_{h}^{-1}\right) M_{\mathrm{th}}\right)+\right. \\
\left.\left.+\alpha_{2}\left(\prod_{h=p}^{n} S_{h}^{2}\right)+\alpha_{1} \sum_{l=p}^{n} \beta_{\imath \jmath}\left(\prod_{h=\jmath+1}^{n} S_{h}^{2}\right)\right)\left(\prod_{h=p}^{j}\left(S_{h}+S_{h}^{-1}\right) M_{\mathrm{th}}\right)\right] .
\end{gathered}
$$

If $p=0$ and $H=T=C_{-u}$, the expression (3.21) takes the form

$$
\| \mathcal{W}_{n+1}\left(B_{1}\left(i_{-w}\right)-\mathcal{W}_{n+1.2}\left(B_{1}\left(i_{-w}\right) \| \leq \rho_{n, \imath}\right.\right.
$$

where

$$
\begin{aligned}
\rho_{n, \imath} & =2^{n+1} I_{n 0} g\left(\prod_{h=0}^{n} b_{h}\right)\left[\gamma_{\imath, n+1}\left(\prod_{h=0}^{n}\left(S_{h}+S_{h}^{-1}\right) M_{\mathrm{v} h}\right)+\right. \\
& \left.\left.+\sum_{\jmath=0}^{n} \beta_{i \jmath}\left(\prod_{h=\jmath+1}^{n} S_{h}^{2}\right)\right)\left(\prod_{h=0}^{j}\left(S_{h}+S_{h}^{-1}\right) M_{\imath h}\right)\right]
\end{aligned}
$$

If we consider $H=\mathcal{X}_{-w}, T=\mathcal{X}_{-w}$, from (2.17), (3.1) and (3.5) for $t \in[p w,(p+1) w]$. it follows that

$$
\left\|\mathcal{X}_{-w}-\mathcal{X}_{i,-w}^{\prime}\right\| \leq\left(\|G(0)\|+\left\|G^{\prime}(0)\right\|\right) E_{\imath, p-1},
$$

and from (3.21) we can write

$$
\left\|\mathcal{W}_{k}\left(B_{1} \mathcal{X}_{-w}\right)-\mathcal{W}_{k, 2}\left(B_{1}, \mathcal{X}_{i,-w}^{\prime}\right)\right\| \leq Y_{n, 2}
$$

where

$$
\begin{gathered}
Y_{n, \imath}=2^{n-p} I_{n p}\left(\|G(0)\|+\left\|G^{\prime}(0)\right\|\right)\left(\prod_{h=p}^{n} b_{h}\right) \\
{\left[\left(S_{p}+S_{p}^{-1}\right) \gamma_{\imath, n+1}\left(\prod_{h=p}^{n}\left(S_{h}+S_{h}^{-1}\right) M_{\imath h}\right)+E_{\imath, p-1}\left(\prod_{h=p}^{n} S_{h}^{2}\right)+\right.} \\
\left.\left.+\left(S_{p}+S_{p}^{-1}\right) \sum_{\jmath=p}^{n} \beta_{\imath}\left(\prod_{h=\jmath+1}^{n} S_{h}^{2}\right)\right)\left(\prod_{h=p}^{j}\left(S_{h}+S_{h}^{-1}\right) M_{\imath h}\right)\right] .
\end{gathered}
$$

Taking $H=(\mathcal{W} F)_{-w}, T=\left(\mathcal{W}^{\imath} F\right)_{-w}$ and using that $t_{p} \in[p w,(p+1) w]$, from (3.13) and (3.17), it follows that

$$
\begin{gathered}
\left\|(\boldsymbol{W} F)_{-w}-\left(\mathcal{W}^{\imath} F\right)_{-w}\right\| \leq \\
\leq \int_{0}^{t-w}\left\|K(t-w, x)-K_{\imath}(t-w, x)\right\|\|F(x)\| d x \leq \\
\leq \int_{0}^{t-w} \gamma_{\iota, p-1} f_{p-1} d x \leq p w \gamma_{\imath, p-1} f_{p-1},
\end{gathered}
$$

and from (3.21) and (3.17),

$$
\begin{gathered}
\left\|\left(\mathcal{W}^{\imath} F\right)_{-w}\right\| \leq \int_{0}^{t-w}\left\|K_{\imath}(t-w, x)\right\|\|F(x)\| d x \leq \\
\leq p w\left(S_{p-1}+S_{p-1}^{-1}\right) M_{\imath, p-1} f_{p-1} .
\end{gathered}
$$

From (3.21) and (3.22) we can write

$$
\left\|\mathcal{W}_{n}\left(B_{1}(\mathcal{W} F)_{-w}\right)-\mathcal{W}_{n, i}\left(B_{1}\left(\mathcal{W}^{\imath} F\right)_{-w}\right)\right\| \leq I_{n, i},
$$


where

$$
\begin{gathered}
U_{n, 2}=2^{n-p+1} I_{n p} p w f_{p-1}\left(\prod_{h=p}^{n} b_{h}\right) \\
{\left[\left(S_{p-1}+S_{p-1}^{-1}\right) M_{\imath, p-1} \gamma_{\imath, n+1}\left(\prod_{h=p}^{n}\left(S_{h}+S_{h}^{-1}\right) M_{\imath h}\right)+\gamma_{t, p-1}\left(\prod_{h=p}^{n} S_{h}^{2}\right)+\right.} \\
\left.\left.+\left(S_{\gamma^{\prime}-1}^{\prime}+S_{p-1}^{-1}\right) M_{i, p-1} \sum_{J=p}^{n} \beta_{\imath \jmath}\left(\prod_{h=\jmath+1}^{n} S_{h}^{2}\right)\right)\left(\prod_{h=p}^{j}\left(S_{h}+S_{h}^{-1}\right) M_{\imath h}\right)\right] .
\end{gathered}
$$

If we denote by $X_{1}(t)$ for $i \geq \iota_{0}$ the approximate solution of $(1.1)$ defined in $[n u,(n+1) w]$ by

$$
\begin{gathered}
X_{\imath}=\mathcal{X}_{\imath}+\mathcal{W}^{i} F+\sum_{k=1}^{n}(-1)^{k} \mathcal{W}_{k \imath}\left(B_{1}\left(\mathcal{W}^{i} F\right)_{-u}\right)+ \\
+\sum_{k=1}^{n}(-1)^{k} \mathcal{W}_{k \imath}\left(B_{1}\left(\mathcal{X}_{\imath}^{\prime}\right)_{-w}\right)+(-1)^{n+1} \mathcal{W}_{n+1, i}\left(B_{1} G_{-w}\right),
\end{gathered}
$$

from $(2.20),(3.23)$ and the previous comments of Section 3, it follows that the error $X^{\prime}(l)-X_{2}(l)$ of the approximation $X_{2}(t)$ with respect to the exact solution $X^{\prime}(t)$ of $(1.1)$, for $1 \geq 1_{0}$ and $t \in$ $[n w,(n+1) w]$ is bounded above by

$$
\begin{gathered}
\left\|X(t)-X_{\imath}(t)\right\| \leq \\
\leq\left(\|G(0)\|+\left\|G^{\prime \prime}(0)\right\|\right) E_{\imath, n}+(n+1) v f_{n} \gamma_{\imath, n}+\rho_{\imath, n}+\sum_{h=1}^{n}\left(l_{k, 2}+Y_{k, 2}\right) .
\end{gathered}
$$

Thus, for a fixed interval $\left[n_{0} w,\left(n_{0}+1\right) w\right]$ and an admissible error c, to construct a finite analytic approximate solution whose error be smaller than $\epsilon$ in $\left[n_{0} w,\left(n_{0}+1\right) w\right]$ it is sufficient to take $\imath \geq i_{0}$ such that

$$
\left(\|G(0)\|+\left\|G^{\prime}(0)\right\|\right) E_{\imath, n}+(n+1) w f_{n} \gamma_{\imath, n}+\rho_{\imath, n}+\sum_{k=1}^{n}\left(U_{k, \imath}+Y_{k, \imath}\right)<\epsilon .
$$

Hence the following result has been proved.

THEOREM 3. Let us consider the problem (1.1) under the hypotheses of Theorem 2 and let, us use the previous notation. If $i_{0}$ is the first positive integer $i$ satisfying $(3.8)$ and $X_{3}(t)$ is the function defined by (3.23) for $i \geq i_{0}$, then $X_{2}(t)$ converges to the exact solution $X^{\prime}(t)$ of $(1.1)$ as $i \rightarrow+\infty$, for any $t>0$. If $n_{0}$ is a positive integer, then the error of the approximate solution $X_{1}(t)$ with respect to the exact solution $X(t)$ satisfies (3.24) for $t \in\left[n_{0} w,\left(n_{0}+1\right) w\right]$ and $i \geq i_{0}$. Given an admissible error $\epsilon>0$, taking $i \geq i_{0}$ satisfying (3.25), one gets an approximation whose error is bounded above by $\epsilon$ for $t \in\left[n_{0} w,\left(n_{0}+1\right) w\right]$.

ACKNOWLEDGEMENTS: This work has been supported by Generalidad Valenciana grant from the I.V.E.I. and D.G.I.C.Y.T. grant PS90-0140.

\section{References}

1. R. BELLMAN and K. L. COOKE, Differential-Difference Equations, Academic Press, New York, 1962.

2. V. B. CHEREPENNIKOV, Investigation of the solutions of a class of differential-functional equations, Sb. Asymptotic Methods in System Dynamics, Ed. USF Siberian Filial Arad. Sci., Irkutsk (1985), 38-44 (in Russian).

3. R. D. DRIVER, Ordinary and Delay Differential Equations, Applied Mathematics Sciences 20, Springer, Berlin, 1977.

4. T. M. FLETT, Differential Analysis, Cambridge Univ. Press, 1980. 
5. G. GOLUB and ('. F. VAN LOAN, Matrix Computations, Johns Hopkins Univ. Press, Baltimore, MA., 198:3.

6. I. S. GRADSHTEYN and I. M. RYZHIh, Table of Integrals, Series and Products, Academic Press, New York, 1980.

7. L. JODAR and M. LEGUA, Solving second order matrix differential equations with regular singular points avoiding the increase of the problem dimension, Appl. Math. Comput. 5;3 (1993), 191-206.

8. L. JODAR and J. A. MARTIN, Explicit solution of systems of second order differential difference equations, J. Comput. Appl. Maths., 43 (1992), 323-334.

9. L. JODAR and J. A. MARTIN, Analytical solution of a class of coupled second order differentialdifference equations, Internat. J. Math. \& Math. Sci., Vol. 16 No. 2 (1993) 385-396.

10. J. K. HALE, Theory of Functional Differential Equations, Springer Verlag, 1977, New York.

11. G. I. MARCHUK, Mathematical Models in Immunology, Translation Series, Optimization Software, Springer, New York, 1983.

12. A. D. MYSHKIS, Linear Differential Equations with a Retarded Argument, Ed. Gostekhizdat, Moscow-Leningrad, 1951 (in Russian; 2nd Edit. 1972).

13. M. OKAMOTO and K. HAYASHI, Frequency conversion mechanism in enzymatic feedback systems, J. Theor. Biol. 108 (1984), 529-537.

14. J. M. ORTEGA, Numerical Analysis, A second course, Academic Press, New York, 1972.

15. E. PINNEY, Ordinary Difference-Differential Equations, Univ. of California Press, Berkeley, Los Angeles, 1958.

16. V. I. RODINOV, Analytic solution of a linear functional differential equation with a linear deviation of the argument, Differential equations, 25 (1989), No. 4, 616-626 (in Russian). 


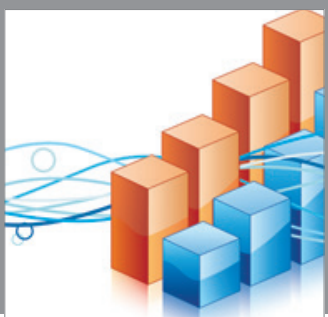

Advances in

Operations Research

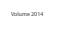

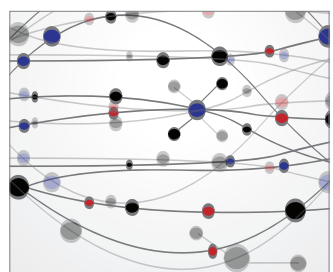

\section{The Scientific} World Journal
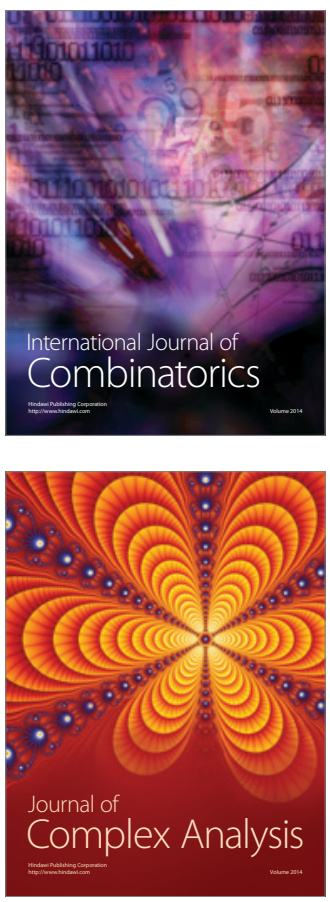

International Journal of

Mathematics and

Mathematical

Sciences
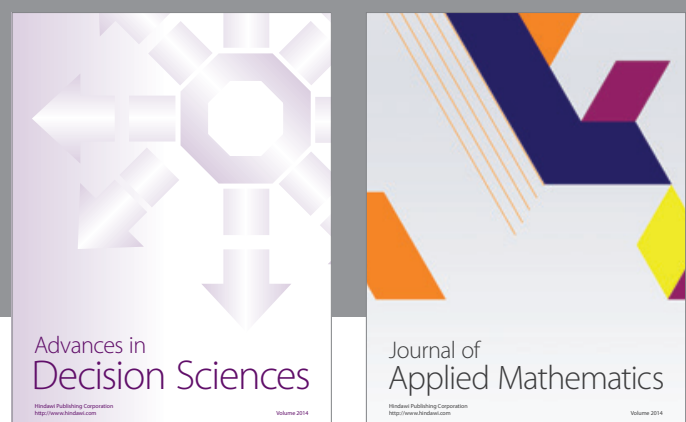

Journal of

Applied Mathematics
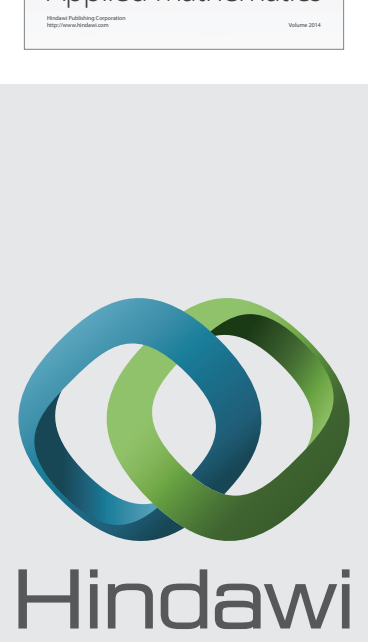

Submit your manuscripts at http://www.hindawi.com
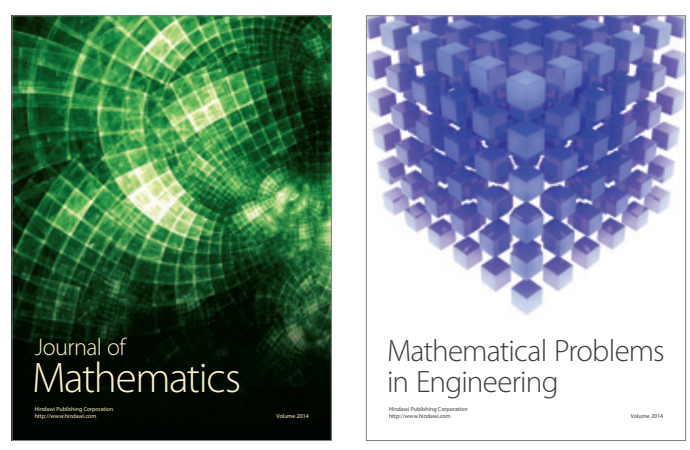

Mathematical Problems in Engineering
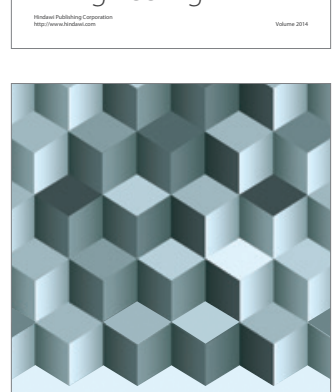

Journal of

Function Spaces
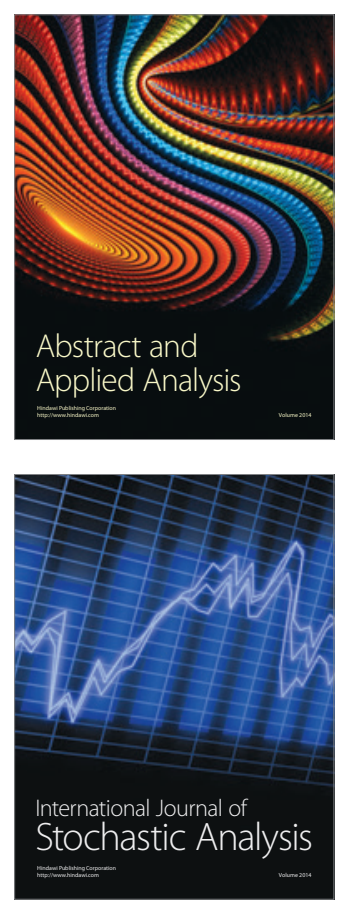

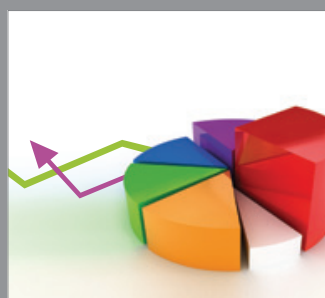

ournal of

Probability and Statistics

Promensencen
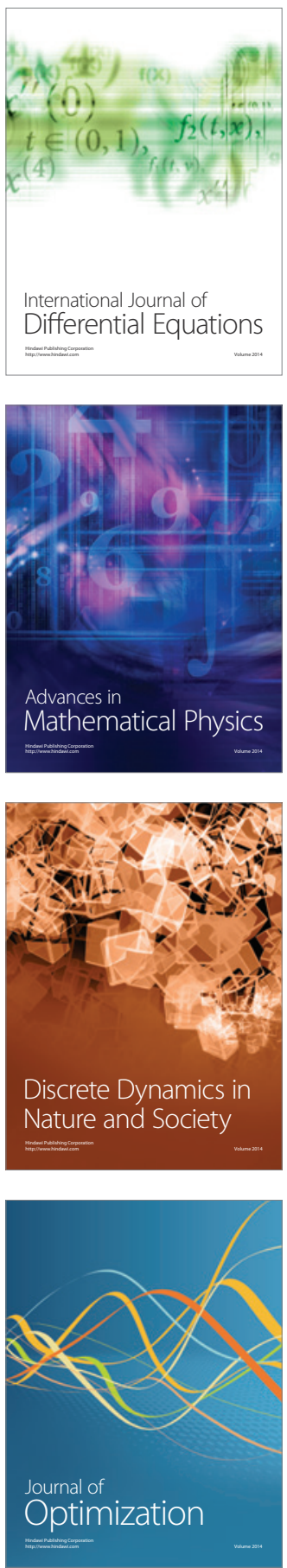